\title{
Toll-like Receptor 2 Polymorphism Is Associated With Preterm Birth
}

\author{
TANNETTE G. KREDIET, SELMA P. WIERTSEMA, MARJOLEIN J. VOSSERS, SANNE B.E.A. HOEKS, \\ ANDRÉ FLEER, HENK J.T. RUVEN, AND GER T. RIJKERS
}

\begin{abstract}
Departments of Neonatology [T.G.K., S.B.E.A.H.] and Pediatric Immunology [S.P.W., M.J.V., S.B.E.A.H., G.T.R.], Wilhelmina Children's Hospital, University Medical Center Utrecht, 3508 AB, The Netherlands; Eijkman Winkler Center for Microbiology, Infectious Diseases and Inflammation [A.F.], University Medical Center Utrecht, The Netherlands; Medical Microbiology and Immunology [H.J.T.R., G.T.R.], St. Antonius Hospital, 3435 CM, The Netherlands
\end{abstract}

\begin{abstract}
Evidence is increasing for a role of polymorphisms in maternal or fetal innate immune response genes in preterm birth. Toll-like receptors (TLRs) are important receptors in the innate immunity. The genotype distribution of two TLR2 single nucleotide polymorphisms (SNPs) and one TLR4 SNP were determined among 524 neonates and associated with gestational age (GA). Genomic DNA was isolated from prospectively collected blood samples and polymorphisms in TLR2 (T-16934A, RS4696480 and Arg753Gln, RS5743708) and TLR4 (Thr399Ile, RS4986791) were determined using sequence specific primers by PCR. Allele frequencies of two TLR2 SNPs and one TLR4 SNP were analyzed according to prematurity. Analysis among 305 infants, after exclusion of infants born after multiple pregnancy or because of preeclampsia, revealed significantly shorter GAs for infants carrying two polymorphic TLR2 alleles (-16934TA/AA and 753ArgGln/GlnGln) compared with infants carrying one polymorphic and one wild-type allele or two wild-type alleles (median GA 30.6 wk versus 34.1-36.8 wk, respectively, $p<0.02$ ). Carriage of two variant TLR2 alleles potentially leads to aberrant innate immune responses, which may have contributed to very preterm birth. (Pediatr Res 62: 474-476, 2007)
\end{abstract}

$\mathrm{P}$ reterm birth remains a major public health concern because of its associated perinatal morbidity and mortality (1). Evidence is increasing of an important role of polymorphisms in maternal or fetal immune response genes in preterm birth $(2,3)$. Several single nucleotide polymorphisms (SNPs) in immune response genes, particularly in proinflammatory cytokine genes, have been investigated for their contribution to the etiology of preterm birth (2-5). The innate immune system's rapid recognition of pathogens is mediated through toll-like receptors (TLRs) and binding of a microbe to a TLR leads to activation of several inflammatory pathways (6-9). SNPs in TLR genes may affect TLR function, contributing to a modulated inflammatory response. Up-regulation of TLRs may be one of the mechanisms by which intrauterine inflammation signals parturition and triggers preterm labor (10). This is supported by the fact that the majority of pregnancies that end with very preterm birth are more likely to be complicated by chorioamnionitis and/or neonatal infection acquired by

Received November 30, 2006; accepted May 16, 2007.

Correspondence: Tannette G. Krediet, M.D., Department of Neonatology, Room KE.04.123.1, Wilhelmina Children's Hospital, University Medical Center, P.O. Box 85090, 3508 AB Utrecht, The Netherlands; e-mail: t.krediet@umcutrecht.nl vertical transmission from the mother $(11,12)$. Moreover, an increased frequency of two TLR4 polymorphisms has been found in a population of preterm Finnish infants when compared with term control infants (5). The most important TLR4 ligand is LPS from Gram-negative microorganisms.

We hypothesize that polymorphisms in the TLR2 and TLR4 genes result in modulation of fetal inflammatory responses, which trigger preterm birth. To address this issue, we determined the genotype distribution of two common TLR2 (T-16934A, Arg753Gln) and two cosegregating TLR4 (Asp299Gly and Thr399Ile) SNPs among 524 infants admitted to the neonatal intensive care unit of our hospital and associated this with gestational age (GA).

\section{METHODS}

Patient population. In a prospective study, we separately stored a portion of the whole blood sample collected for routine laboratory analyses of 524 infants admitted to the neonatal intensive care unit of our tertiary care hospital (Wilhelmina Children's Hospital, University Medical Center, Utrecht, The Netherlands) between 2002 and 2004. Clinical data of the infants and their mothers were collected prospectively. Infants born preterm due to the condition of mothers with preeclampsia or multiple pregnancy were excluded because they are confounding. The study was approved by the Medical Ethics Committee of the University Medical Center Utrecht. Informed parental consent was obtained from all participating subjects.

DNA isolation and SNP genotyping. Genomic DNA was isolated from whole blood using a Qiaamp DNA blood kit (Qiagen, Hilden, Germany).

Polymorphisms in TLR2 [T-16934A (RS4696480) and Arg753Gln (RS5743708)] and TLR4 [Thr399Ile (RS4986791)] were determined using sequence specific primers and polymerase chain reaction (SSP-PCR) technique (13). After SSP-PCR, samples were loaded on a $2 \%$ agarose gel containing ethidium bromide, electrophoresed for $15 \mathrm{~min}$ at $300 \mathrm{~V}$, and visualized under ultraviolet light. The TLR4 Asp299Gly polymorphism (RS4986790) was determined using a TaqMan genotyping assay.

Statistical analyses. The median GA according to combinations of the TLR2 SNPs was compared using one-way analysis of variance (ANOVA). Data analyses were performed using SPSS version 12.0.1 for Windows (SPSS Inc., Chicago, IL). $P$ values $<0.05$ were considered statistically significant.

\section{RESULTS}

The relevant clinical characteristics of the 524 infants are shown in Table 1. Because preeclampsia and multiple pregnancies are important risk factors for prematurity, we excluded infants born of mothers with these conditions. There-

Abbreviations: GBS, group B streptococcus; SNP, single nucleotide polymorphism; TLR, toll-like receptor 
Table 1. Clinical characteristics of all study subjects $(\mathrm{n}=524)$ and of 305 infants, after exclusion of infants born in multiple pregnancies or after preeclampsia

\begin{tabular}{lcc}
\hline \multicolumn{1}{c}{ Total no. of patients } & All $(n=524)$ & Multiple birth and eclampsia excluded $(n=305)$ \\
\hline Male/female, $n$ & 314 & 191 \\
Birth weight, g, $(95 \% \mathrm{CI})$ & $1958.2(1873.6-2042.8)$ & $2324.6(2207.3-2441.9)$ \\
Gestational age, wk $(95 \% \mathrm{CI})$ & $33.2(32.8-33.6)$ & $34.5(33.9-35.0)$ \\
Term: $\geq 37 \mathrm{wk}, n(\%)$ & $117(22.3)$ & $105(34.4)$ \\
Moderately preterm: $32-37 \mathrm{wk}, n(\%)$ & $143(27.3)$ & $81(26.6)$ \\
Very preterm: $<32 \mathrm{wk}, n(\%)$ & $264(50.4)$ & $119(39.0)$ \\
Infants born from multiple pregnancies, $n(\%)$ & $125(23.9)$ & Excluded \\
Preeclampsia, $n(\%)$ & $108(20.6)$ & Excluded \\
Preterm premature rupture of membranes, $n(\%)$ & $58(11.1)$ & $49.1)$ \\
Early-onset sepsis, $n(\%)$ & $11(2.1)$ & $9(3.0)$ \\
\hline
\end{tabular}

Table 2. Allele frequencies of the TLR2-16934 (RS4696480), TLR2 2285 (RS5743708), and TLR4 8851 (RS4986791) SNPs in the total study group and in subgroups

\begin{tabular}{|c|c|c|c|}
\hline & \multicolumn{2}{|c|}{ TLR2 } & \multirow{2}{*}{$\frac{\text { TLR4 }}{8851 \mathrm{C} / \mathrm{T}(\mathrm{RS} 4986791)}$} \\
\hline & $-16934 \mathrm{~A} / \mathrm{T}(\mathrm{RS} 4696480)$ & 2258 G/A (RS5743708) & \\
\hline All $(n=524 ; \%)$ & $517 / 531(49.3 / 50.7)$ & $1019 / 29(97.2 / 2.8)$ & $992 / 56(94.7 / 5.3)$ \\
\hline Preterm premature rupture of membranes $(n=58 ; \%)$ & $57 / 59(49.2 / 50.8)$ & $112 / 4(96.6 / 3.4)$ & $110 / 6(94.8 / 5.2)$ \\
\hline Preeclampsia $(n=108 ; \%)$ & $109 / 107(50.5 / 49.5)$ & $205 / 11(94.9 / 5.1)$ & $204 / 12(94.4 / 5.6)$ \\
\hline Born in multiple pregnancy $(n=125 ; \%)$ & $121 / 129(48.4 / 51.6)$ & $241 / 9(96.4 / 3.6)$ & $233 / 17(93.2 / 6.8)$ \\
\hline Preeclampsia or multiple pregnancies excluded $(n=305 ; \%)$ & $298 / 312(48.9 / 51.1)$ & $596 / 14(97.7 / 2.3)$ & $581 / 29(95.2 / 4.8)$ \\
\hline
\end{tabular}

fore, the statistical analysis for a possible association between TLR polymorphisms and prematurity was performed in 305 of the 524 infants.

The allele frequencies of the examined TLR2 and TLR4 SNPs were in Hardy-Weinberg equilibrium (Table 2). Because the TLR4 Asp299Gly and Thr399Ile TLR4 SNP were totally cosegregating, we analyzed one SNP (Thr399Ile).

The median GA of infants was similar across the individual TLR2 and TLR4 genotypes. However, comparing the median GA of infants being wild type on both TLR2 positions, infants carrying one polymorphic allele and infants carrying two polymorphic alleles showed that the infants with a double polymorphism in the TLR2 allele had more than 3 wk shorter GA

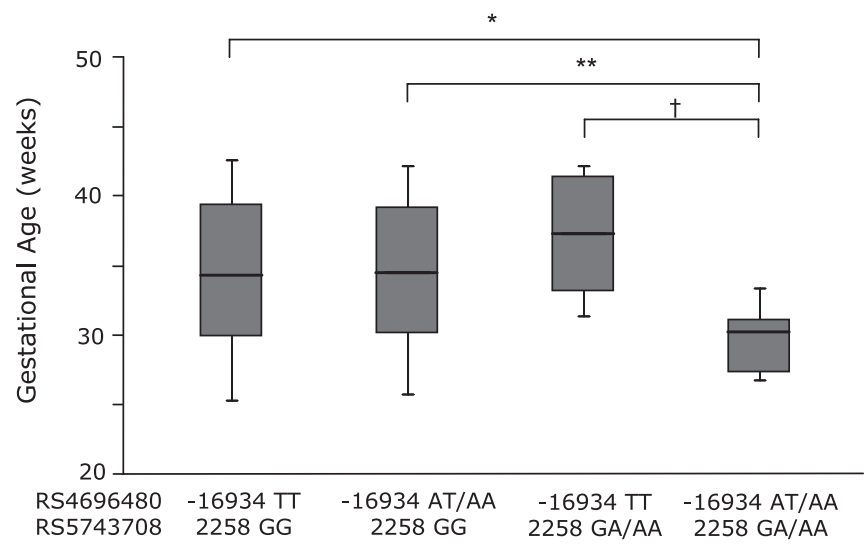

Figure 1. Median GA according to polymorphisms in the TLR2 gene. Infants born in multiple pregnancy or preeclampsia were excluded. Data are grouped according to - 16934 and 2258 genotype: -16934 TT $2258 \mathrm{GG}, n=79 ;-16934$ AT/AA 2258GG, $n=212$; -16934 TT $2258 \mathrm{GA} / \mathrm{AA}, n=7,-16934 \mathrm{AT} / \mathrm{AA}$ $2258 \mathrm{GA} / \mathrm{AA}, n=7$. Median values are indicated by a bar, the boxes represent the 25th-75th percentile and the whiskers minimum and maximum; $p$ values as calculated by one-way ANOVA are indicated in the figure. ${ }^{*} p=$ $0.016 ; * * p=0.009 ; \dagger p=0.003$. (median GA 30.6 wk in the latter group versus 34.1-36.8 wk in the other groups; $p<0.02$ ) (Fig. 1). The polymorphism in TLR4 did not contribute to GA.

\section{DISCUSSION}

The present study showed a significant association between the carriage of two TLR2 polymorphic alleles and very preterm birth. Carriage of only one TLR2 polymorphic allele did not influence the duration of gestation.

A study in a Finnish population by Lorenz et al. (5) found a trend toward a higher frequency of TLR2 polymorphisms (TLR Arg753Gln) in preterm infants compared with term infants, but this association was not significant. TLR2 predominantly responds to Gram-positive bacteria, such as group B streptococci (GBS), the most frequent causative microorganism for neonatal early-onset sepsis. However, we were not able to establish an association between TLR2 mutations and GBS sepsis, probably due to the very low incidence of cultureproven early-onset GBS sepsis (14). Elovitz and colleagues (10) studied the involvement of TLRs in preterm parturition using a mouse model with localized intrauterine inflammation and found that lipopolysaccharide stimulates both TLR4 and TLR2 mRNA expression in the uterus, cervix, and placenta, resulting in an exaggerated host response to microorganisms colonizing the vagina, such as GBS. However, whether the enhanced maternal host response through activated TLRs, or, alternatively but not mutually exclusively, a modulated fetal host response through polymorphisms in TLRs is prevalent cannot be concluded yet. Studies among larger cohorts are needed to confirm the hypothesis that fetal TLR2 polymorphisms contribute to the development of Gram-positive bacterial infections in the perinatal period.

The TLR2 SNPs used in this study are not tagging SNPs for this gene but were chosen because of the functional conse- 
quences. The TLR2 gene does, however, reside in a large block of LD on chromosome 4.

An important issue for the interpretation of our data is the varying distribution of innate immunity genes among populations of different ethnic origin (15). However, although we were not informed on the exact ancestral origin of our study population, the vast majority of our neonatal intensive care unit population is white, and therefore ethnicity was not considered a confounding factor.

Associations between polymorphisms in other maternal genes and preterm birth have been described: SNPs in tumor necrosis factor, interleukin (IL)-1 $\beta$ receptor antagonist, IL-4, matrix metalloproteinase $9, \beta_{2}$-adrenergic receptor, vascular endothelial growth factor, and factor $\mathrm{V}$ Leiden are all associated with preterm delivery (4). Therefore, both maternal and fetal polymorphisms in inflammatory pathway genes should be investigated for their impact on preterm birth.

In conclusion, our study suggests that carriage of two variant TLR2 alleles potentially leads to an aberrant fetal innate immune response, contributing to very preterm birth. Additional studies involving polymorphisms of both maternal and fetal innate immunity genes may further substantiate the importance of these genes as risk factors for intrauterine complications and preterm birth.

Acknowledgments. The authors thank Dr. Coline van Moorsel, Department of Pulmonology, St. Antonius Hospital, Nieuwegein, The Netherlands, for help with genetic analysis.

\section{REFERENCES}

1. Slattery MM, Morrison JJ 2002 Preterm delivery. Lancet 360:1489-1497

2. Crider KS, Whitehead N, Buus RM 2005 Genetic variation associated with preterm birth: a HuGE review. Genet Med 7:593-604

3. Varner MW, Esplin MS 2005 Current understanding of genetic factors in preterm birth. BJOG 112:28-31

4. Härtel Ch, Finas D, Ahrens P, Kattner E, Schaible T, Muller D, Segerer H, Albrecht K, Moller J, Diedrich K, Gopel W. Genetic Factors in Neonatology Study Group 2004 Polymorphisms of genes involved in innate immunity: association with preterm delivery. Mol Hum Reprod 10:911-915

5. Lorenz E, Hallman M, Marttila R, Haataja R, Schwartz DA 2002 Association between the Asp299Gly polymorphisms in the Toll-like receptor 4 and premature births in the Finnish population. Pediatr Res 52:373-376

6. Janeway CA Jr, Medzhitov R 2002 Innate immune recognition. Annu Rev Immunol 20:197-216

7. Akira S, Takeda K 2004 Toll-like receptor signalling. Nat Rev Immunol 4:499-511

8. Aderem A, Ulevitch RJ 2000 Toll-like receptors in the induction of the innate immune response. Nature 406:782-787

9. Beutler B, Hoebe K, Du X, Ulevitch RJ 2003 How we detect microbes and respond to them: the Toll-like receptors and their transducers. J Leukoc Biol 74:479-485

10. Elovitz MA, Mrinalini C 2005 Can medroxyprogesterone acetate alter Toll-like receptor expression in a mouse model of intrauterine inflammation? Am J Obstet Gynecol 193:1149-1155

11. Goldenberg RL, Hauth JC, Andrews WW 2000 Intrauterine infection and preterm delivery. N Engl J Med 342:1500-1507

12. Goldenberg RL, Rouse DJ 1998 Prevention of premature birth. N Engl J Med 339:313-320

13. Bunce M, O’Neill CM, Barnardo MC, Krausa P, Browning MJ, Morris PJ, Welsh KI 1995 Phototyping: comprehensive DNA typing for HLA-A, B, C, DRB1, DRB3, DRB4, DRB5 \& DQB1 by PCR with 144 primer mixes utilizing sequence- specific primers (PCR-SSP). Tissue Antigens 46:355-367

14. Stoll BJ, Hansen N, Fanaroff AA, Wright LL, Carlo WA, Ehrenkranz MD, Lemons JA, Donovan EF, Stark AR, Tyson JE, Oh W, Bauer CR, Korones SB, Shankaran S, Laptook AR, Stevenson DK, Papile L-A, Poole W 2002 Changes in pathogens causing early-onset sepsis in very-low-birth-weight infants. N Engl J Med 347:240247

15. Nguyen DP, Genc M, Vardhana S, Babula O, Onderdonk A, Witkin SS 2004 Ethnic differences of polymorphisms in cytokine and innate immune system genes in pregnant women. Obstet Gynecol 104:293-399 